\section{$\underset{\substack{\text { hommes } \\ \text { \& migrations }}}{ }$}

\section{Hommes \& migrations}

Revue française de référence sur les dynamiques

migratoires

$1322 \mid 2018$

Exposer les migrations

\title{
Moving Beyond Borders : la fabrique d'une œuvre scientifique
}

Marie Arlais, Lucie Bacon, Olivier Clochard et Raphaël Rialland

\section{(2) OpenEdition}

\section{Journals}

\section{Édition électronique}

URL : https://journals.openedition.org/hommesmigrations/6701

DOI : 10.4000/hommesmigrations. 6701

ISSN : 2262-3353

\section{Éditeur}

Musée national de l'histoire de l'immigration

\section{Édition imprimée}

Date de publication : 1 juillet 2018

Pagination : 125-134

ISBN : 978-2-919040-42-1

ISSN : $1142-852 X$

\section{Référence électronique}

Marie Arlais, Lucie Bacon, Olivier Clochard et Raphaël Rialland, « Moving Beyond Borders : la fabrique d'une œuvre scientifique », Hommes \& migrations [En ligne], 1322 | 2018, mis en ligne le 01 juillet 2020, consulté le 21 janvier 2022. URL : http://journals.openedition.org/hommesmigrations/6701 ; DOI : https://doi.org/10.4000/hommesmigrations.6701 


\title{
MOVING BEYOND BORDERS: LA FABRIQUE D'UNE CEUVRE SCIENTIFIQUE
}

Par MARIE ARLAIS, artiste et documentariste, collectif Étrange miroir (Nantes), LUCIE BACON, géographe, doctorante, Migrinter, université de Poitiers/Telemme, Aix-Marseille université, OLIVIER CLOCHARD, géographe, Migrinter, université de Poitiers et RAPHAËL RIALLAND, artiste, musicien et scénographe, collectif Étrange miroir (Nantes).

\author{
Fruit de la collaboration entre le réseau Migreurop et le collectif \\ de scénographes et d'artistes Étrange miroir, l'exposition \\ Moving Beyond Borders propose une exploration des parcours \\ des migrants et des dispositifs responsables de leur errance, \\ dans le Sahara, en mer Méditerranée et/ou aux frontières \\ orientales de l'Union européenne. Le défi de rendre accessible \\ au plus grand nombre dix années de recherche a suscité \\ une approche multimédia des réalités migratoires. \\ Cartes interactives, photographies ou paysages sonores \\ nourrissent ainsi une compréhension sensible du vécu \\ des migrants pour lutter contre les préjugés dont ils sont \\ la cible.
}

Faire évoluer les regards sur les migrations passe par la multiplication des lectures du phénomène et donc par la diversification des mises en scène $e^{1}$. La prolifération de certaines expositions sur les migrations révèle une volonté commune - dans la diversité ! - de comprendre d'abord, d'exprimer un sentiment de révolte ensuite, pour mieux agir enfin.L'une d'entre elles, Moving Beyond Borders, s'articule autour de la rencontre entre deux « univers » qui s'unissent pour porter un message à travers l'élaboration d'une œuvre. Conçue comme une expérimentation scientifique et artistique, cette démarche se traduit par la mise en scène physique et sensible du travail cartographique développé par le réseau Migreurop, tout en cherchant à rendre compte de l'histoire et de l'évolution des politiques migratoires. Les contributions des militants, des chercheurs et des artistes, à la croisée de la pratique scientifique et de la recherche esthétique, tracent ici un trait d'union entre la recherche et la création, et proposent une lecture subtile du phénomène. À quatre voix - celles d'une partie des fabricants de Moving Beyond Borders ${ }^{2}$-, nous proposons de partager ici le cheminement qui a nourri lélaboration de cette exposition. 


\section{Migreurop : des camps au prisme d'un réseau militant et scientifique (Olivier Clochard, Migreurop)}

L'idée d'utiliser la cartographie pour mettre en lumière les évolutions des politiques migratoires européennes et leurs conséquences sur la vie des personnes en situation de migration est apparue dès les premières rencontres des militants et des chercheurs engagés dans la défense des droits des exilés, de ce qui allait devenir le réseau Migreurop. Après le forum social européen de Florence en novembre 2002 (" moment fondateur »), deux rencontres sont organisées à Bruxelles (février et juin 2003) au cours desquelles l'idée est actée de " donner à voir une réalité qu'aucune statistique nationale ou européenne ne permettait d'appréhender : la place que l'enfermement administratif et toutes les formes de confinement des étrangers occupaient, de façon croissante, dans la politique migratoire de l'Union européenne (UE) et de ses États membres, avec le cortège de violations des droits et de violences physiques et psychiques que cela entraînait ${ }^{3}$ ». Cette carte, rendue publique en novembre 2003 lors du Forum social de Paris-Saint-Denis, sera reprise de nombreuses et diverses manières, et deviendra en quelque sorte «la carte de visite » du réseau. Traduite en différentes langues, elle est imprimée autant sur des flyers militants que dans des ouvrages scientifiques. Elle s'accroche en format réduit sur les murs de squats, mais peut également se dévoiler dans de plus grandes dimensions - au grand air - dans les rues de villes européennes (Berlin, Bruxelles,
Copenhague, Paris, Syracuse $\left.{ }^{4}\right)$. Cette carte, dont la sixième version a été produite en 2016, a été diffusée sur de nombreux supports, et rappelle à sa façon que des hommes, des femmes et des enfants sont enfermés au sein des États membres et des pays voisins de l'UE, pour des durées allant de quelques jours à plusieurs mois, voire des années, pour le seul fait de ne pas avoir de visa ou de titre de séjour en règle. Le développement de ces «métastases $^{5}$ » s'opère au cours des années 1980 , période à laquelle commencent à être mis en place dans plusieurs pays européens des centres de rétention administrative qui, près de quarante ans plus tard, constituent un autre archipel s'étendant bien audelà des frontières de l'Europe ${ }^{6}$.

\section{L'essor cartographique au sein des ONG}

Pour chacune des versions de cette carte, la démarche est quasiment la même. Tout d'abord, une dizaine de membres du réseau - voire plus épluche les rapports officiels et associatifs, les sites Internet des institutions (ministères de l'Intérieur, services de l'asile, etc.) pour recueillir des données statistiques selon des critères préétablis ${ }^{7}$; c'est un travail chronophage car les sources demeurent très diverses selon les États ${ }^{8}$. Puis, à partir des tableaux ou des bases de données réalisés, la réflexion est spatialisée à l'échelle européenne pour saisir sur le même plan ces nombreux dispositifs. L'enregistrement de ces premières données ouvre ensuite d'autres voies, car ce que l'on peut voir à une large échelle est souvent différent à un niveau national, régional voire local. À partir de 2011, le

\footnotetext{
3. Migreurop, Atlas des migrants en Europe. Approches critiques des politiques migratoires, Paris, Armand Colin, 2017, p. 164. 4. Ces expositions étaient associées à des moments militants (Emmaüs à Paris, Arci à Syracuse, etc.), mais certaines d'entre elles ont également bénéficié du soutien de diverses institutions (Center for Art on Migration Politics à Copenhague, European Programme for Integration and Migration à Bruxelles, etc.). 5. Expression empruntée à Roger Brunet lorsqu'il décrivait l'archipel des camps du Goulag au sein de l'Union soviétique. Voir Roger Brunet, “ Géographie du Goulag ", in L’Espace géographique, vol. 10, n 3, 1981, p. 218. 6. Ce constat de la multiplication et de la banalisation des camps est aussi au cœur des travaux de Michel Agier. Voir notamment son ouvrage, Un monde de camps, Paris, La Découverte, 2014. 7. Parmi ces critères, il y a la capacité officielle de ces centres, les effectifs annuels des personnes enfermées (hommes, femmes, enfants), les durées maximales de détention, etc. Pour avoir davantage de précisions sur les informations recherchées au sein du réseau, voir la rubrique "Contribuer » du site closethecamps (http://closethecamps.org/contribuer/). 8. Par exemple, pour l'Allemagne, les données - lorsqu'elles sont publiées - sont réparties selon les Länder. Pour l'Ukraine, et pour certaines années, les informations ont été tirées des rapports de la cour des comptes de l'Union européenne. Pour des pays comme la Libye, les témoignages ou les revues de presse sont généralement les seules sources dont nous disposons.
} 
travail des cartographes du réseau se structure pour réaliser les cartes de la seconde édition de l'Atlas des migrants en Europe et la cinquième édition de la carte des camps. En 2013, ce groupe se concentre sur la mise en place d'une "cartographie dynamique de l'enfermement des étranger-e-s », l'objectif étant d'avoir une approche didactique des cartes, de présenter les informations de manière graduelle comme si nous racontions une histoire.

\section{Étrange miroir : les migrations au cœur d'une approche artistique et documentaire (Marie Arlais, Raphael Rialland (Étrange miroir)}

De son côté, le collectif Étrange miroir a été fondé en 2011. Il réalise des projets liant une démarche artistique à des questions sociales et citoyennes. Basé à Nantes, il regroupe une dizaine de personnes aux compétences diverses : musiciens, juriste, graphiste, documentariste, développeur, constructeur, vidéaste, danseur, comédien, etc.

Utilisant la création sonore, musicale et visuelle, ce groupe artistique propose une approche documentaire et sensible des faits sociaux, en particulier sur le thème des migrations et de l'interculturalité. À l'origine, certains des membres étaient bénévoles à la Cimade $^{9}$ et ont œuvré pour le festival Migrant'scène de 2008 à 2011. Dans un contexte où la politique migratoire ne cessait de se durcir, les premières créations se sont appuyées sur des témoignages de militants et de migrants. Ces premières expériences ont nourri le regard et le sens critique : s'intéresser aux phénomènes migratoires, c'est se poser la question de ses frontières personnelles, de son rapport à l'autre. L'iniquité du droit à la mobilité est une réalité frappante. Ce sentiment va bien au-delà du sentiment d'injustice, il nous questionne sur notre rôle, notre place en tant qu'homme né du "bon côté » de la frontière. Cette quête de sens, du pourquoi, nous a encouragés à diversifier nos sources d'inspiration et d'information. Notre intérêt pour la question grandissant, nous avons multiplié les points de vue. Une approche pluridisciplinaire paraît en effet indispensable pour parler des migrations. Le parti pris du collectif est donc d'enrichir sa réflexion avec différentes approches issues des sciences humaines et de la littérature, etc. En miroir de cette démarche, nous avons fait le choix de convoquer différents modes d'expressions artistiques : création sonore, musique, vidéo, animations graphiques, lectures de textes littéraires, danse, scénographie.

Au-delà d'une transmission d'informations, l'acte de mettre en scène une réalité migratoire correspond, en ce qui nous concerne, à un besoin de recourir aux sensations, non pas au sens de rendre "sensationnel ", qui caractérise le traitement médiatique de la question migratoire, de partager au contraire un point de vue sensible, un imaginaire en miroir d'une réalité.

\section{Des collaborations créatrices}

Cependant, la lecture de la question migratoire nécessite des connaissances issues d'un travail mené sur le long terme. Étrange miroir a donc doublé sa démarche artistique d'une volonté de collaboration avec des équipes détentrices d'un savoir scientifique ou d'une expertise de terrain. Nous avons ainsi mené quatre créations en étroite collaboration avec la Cimade (de 2011 à 2014), puis, aujourd'hui avec le réseau Migreurop et l'institut Alliance Europa à Nantes. Cette dynamique de compagnonnages entre artistes et chercheurs vise à trouver ensemble les meilleurs moyens de s'adresser au public, d'interpeller et d'informer.

La principale difficulté, aujourd'hui, est d'attirer l'attention d'un public submergé d'images, de sollicitations et d'informations, notamment sur le web. Dans ce contexte, il peut être pertinent de sortir du virtuel, d'adopter une démarche visant la (re)matérialisation des informations et des connaissances. En revanche, l'outil numérique ne doit pas être perçu comme une menace, mais bien comme un outil complémentaire, pour lequel nous devons 
associer des usages précis. Il s'agit plutôt d'utiliser les technologies numériques et ses possibles, pour expérimenter des créations ludiques et interactives qui donnent envie aux spectateurs, suscitent des questionnements et la volonté d'aller plus loin dans la réflexion.

\section{Moving Beyond Borders, partage d'expériences et convergence d'ambitions (Olivier Clochard, Migreurop)}

Produire une exposition innovante dans sa conception graphique et matérielle, avec des environnements sonores, telle était l'idée nébuleuse que Migreurop aspirait à concrétiser et que le rapprochement avec la compagnie Étrange miroir a permis d'accomplir. À partir de 2013, le réseau Migreurop souhaite diffuser autrement les observations accumulées depuis une dizaine d'années, sur les difficultés que des ressortissants de pays extérieurs à l'UE rencontrent pour voyager, sur les conditions d'enfermement des personnes arrêtées aux frontières et sur les territoires des États, sur les obstacles établis par les autorités qui jalonnent les parcours des migrants (murs, dispositifs biométriques, etc.).

En juin 2014, lors de la troisième édition du festival Téciverdi organisé par la municipalité de Niort dont le thème était consacré aux migrations, Migreurop est sollicité pour exposer des cartes et des photographies. Une partie des cartes réalisées au sein du réseau sont donc sélectionnées, celles de la seconde édition de l'Atlas des migrants en Europe (2012), celles du rapport « La face cachée des camps d'étranger-e-s en Europe $\mathrm{e}^{10}$ » et des cartes qui avaient été retravaillées pour les séminaires de l'Anti-Atlas des frontières ${ }^{11}$. Cinq modules composés de cartes, de photographies ${ }^{12}$ et de textes sont pensés et préfigurent ceux de la future exposition Moving Beyond Borders: l'externalisation des contrôles migratoires (visas, officiers de liaison), les boat people et Frontex, la détention des migrants en Europe et, au-delà des frontières «intelligentes » avec la question de la biométrisation des contrôles, la liberté de circulation. Lors de ce festival, la compagnie Étrange miroir avait été également invitée à venir présenter son spectacle Mother Border. La compagnie avait déjà travaillé avec la Cimade, on l'a dit, association fondatrice et membre de Migreurop. L'idée d'un rapprochement fut discutée lors du festival avant d'être actée autour d'un projet commun.

\section{Outil vidéo, objet atlas et paysages sonores : au cœur de la fabrique (Raphaël Rialland, Étrange miroir)}

Pour Moving Beyond Borders, notre rôle consista à opérer un jeu réflexif à partir de la trame proposée pour faire œuvre commune. Migreurop nous a fourni une grande diversité de données - textes, cartes dessinées à la main ou réalisées avec des logiciels, photographies, enregistrements d'entretiens. Nous avons réuni des compétences pour mettre en forme ce contenu scientifique : concevoir une scénographie, mettre en son, rendre sensible.

Une fois les contenus entre nos mains, il s'agissait dans un premier temps de nous familiariser avec ce qu'ils avaient à raconter, autant sur le plan esthétique qu'informatif, dans l'optique de valoriser leur dimension graphique et d'en proposer une lecture, d'imaginer une narration.

L'utilisation de la vidéo s'est avérée rapidement une piste judicieuse. Elle nous a permis de scénariser les informations et leur ordre d'apparition, de proposer les contenus dans plusieurs langues sans surcharger le champ visuel (chaque carte/film est disponible dans cinq langues). Enfin, nous avons pu créer des animations vidéo proposant une lecture vivante des cartes et des textes qui les accompagnent. 
L'outil vidéo nous a imposé une contrainte technique en nous obligeant à avoir des outils de haute définition (HD). En effet, si la HD donne une sensation de netteté lors de la projection en salle sur grand écran, il en va autrement pour la précision de la restitution de textes, de dessins au trait ou de graphiques à une distance rapprochée. Le choix du medium vidéo nous a donc contraints à faire des choix parfois radicaux en termes de contenus et de mise en page, à utiliser une taille de police minimum pour ce qui concerne les textes, et à supprimer certaines informations contenues initialement dans les cartes.

\section{Inventer des livres qui offrent des histoires en différentes langues}

Mais le fait de sélectionner les informations pour qu'elles soient lisibles correspondait aussi à l'ambition de départ: proposer des contenus scientifiques dans un espace d'exposition, et dans la temporalité d'une visite d'exposition. À la différence d'un lecteur qui consulte un ouvrage, le visiteur est, présume-t-on, dans une disposition à la surprise, dans une situation où il apprécie que l'on le prenne en charge. Il s'agissait donc pour nous d'interpeller le public tout en lui facilitant en quelque sorte la lecture des informations, de sorte qu'il ne puisse pas passer à côté des messages. À l'intention informative s'est donc ajoutée une volonté de raconter des histoires. L'autre entrée importante a été le choix de donner une dimension interactive à l'exposition pour que le visiteur soit également acteur dans le parcours scénographique et que sa curiosité soit attisée par cet aspect ludique.

Nous avons donc conçu des livres-objets dans lesquels sont projetés les textes et les cartes animées lorsque le visiteur en manipule les pages: l'objet «atlas » nous intéressait en tant qu'élément familier faisant référence au domaine de la cartographie. Le visiteur choisit sa langue et est invité à tourner les pages. Les cartes se dessinent et s'animent, les informations sont délivrées de manière progressive, maintenant le spectateur en haleine.

L'immersion est également favorisée par les paysages sonores qui accompagnent chacune des cartes. La création de ces ambiances sonores a été un formidable terrain de jeu créatif. Chacun des artistes sonores a pu réinterpréter librement l'histoire de chaque carte pour poser le climat sonore de ces petits cinémas sur table. Composés de manière à pouvoir se mêler harmonieusement dans l'espace d'exposition, ces paysages sonores tissent un lien physique entre les cinq modules.

\section{Au-delà de la collaboration : construire du commun, bâtir du collectif (Marie Arlais, Étrange miroir)}

Au-delà de la réalisation de l'exposition, nous avons apprécié la dimension collective de l'ensemble du projet. Ce projet a permis d'aménager un espace propice au dialogue, autour d'une question éminemment importante. La richesse de la collaboration entre artistes, militants et chercheurs se situe dans cette rencontre entre rigueur et liberté, utopie et réalité. À l'instar de ce qu'apporte la migration à nos sociétés, cette richesse n'est pas vraiment quantifiable ou mesurable. La construction de ce collectif a suscité ce qu'Édouard Glissant appelle une "pensée du tremblement » qui "résiste aux raidissements des pensées de système et aux emportements des systèmes de pensée. Elle ouvre l'identité sur le rapport à l'Autre et sur le change qui provient alors de l'échange, sans que cette identité en soit perturbée ni dénaturée. Elle est la pensée sismique du monde qui tremble en nous et autour de nous ${ }^{13}$ ». 
Carte réalisée par Lucie Bacon et Nicolas Lambert, in Migreurop, Atlas des migrants en Europe. Approches critiques des politiques migratoires, Paris, Armand Colin, 2017, p. 175.

\section{Exemple d'une expérience collaborative cartographiant l'errance d'un exilé (Lucie Bacon, Migreurop)}

Parmi les dix cartes animées qui sont exposées, nous proposons de détailler ici le cheminement de la création de l'une d'entre elles, afin de saisir la manière dont des matériaux d'enquêtes (récits, dessins, etc.) sont travaillés par différentes personnes pour parvenir à un objet artistique et singulier.

La carte intitulée "Cinq ans pour rejoindre Hambourg depuis Kaboul » retraçant le parcours d'Ahmed ${ }^{14}$ est l'une des cartes qui composent le module 2, consacré à l'enfermement des étrangers sur le territoire de l'Union européenne. Cette carte est associée en binôme à la carte numérique intitulée "L'archipel des camps », réalisée à l’aide de 
logiciels cartographiques et qui figure la présence des camps en Europe et au-delà, la moyenne annuelle du nombre d'étrangers détenus, la durée maximale de détention, ainsi que l'évolution du nombre de camps d'étrangers depuis les années 2000. Complémentaire, la cartographie du parcours d'Ahmed a pour objectif de montrer l'impact de l'enfermement sur la vie d'un individu. Comme toutes les autres cartes dessinées à la main, cette carte vise à retracer un parcours individuel, à travers une approche sensible et personnelle, tout en pointant les dispositifs qui participent à sa complexification. Il fallait donc trouver le récit d'une personne ayant parcouru différents espaces et vécu des expériences multiples de privation de liberté.

Nous avons sollicité Bénédicte Michalon, chercheuse et membre de Migreurop, notamment pour les travaux qu'elle menait au sein du programme Terrferme portant sur les dispositifs contemporains de l'enfermement. Dans ce cadre, elle avait effectué un terrain d'enquête en Roumanie auprès d'étrangers maintenus en rétention administrative ou sortis de ces dispositifs. Elle avait obtenu de la part des autorités roumaines l'autorisation de conduire des entretiens avec diverses personnes rencontrées dans ce contexte. Notre requête lui évoque alors deux récits qu'elle avait précédemment recueillis. Le premier entretien, réalisé avec Violaine Jaussaud, avait déjà permis de réaliser une carte pour l'Atlas des migrants en Europe de Migreurop ${ }^{15}$. Le second, en cours de retranscription, promettait davantage de « rebondissements » et n'avait encore jamais été exploité. Réalisé à Bucarest en 2010, cet entretien permettait de reconstituer le parcours migratoire d'Ahmed, citoyen afghan, depuis son pays d'origine quitté en octobre 2008, jusqu'à l'arrivée en Roumanie en août 2010. Les réseaux sociaux, et notamment Facebook, ont ensuite permis à Bénédicte Michalon et Ahmed de se suivre, et d'apprendre qu'Ahmed était, enfin, arrivé en Allemagne en juillet 2011. Aucun entretien n'ayant été mené sur cette dernière étape du parcours, celleci n'a pu être cartographiée, faute de données suffisantes, mais est signifiée dans le titre de la carte.

\section{Construire la légende et dessiner}

À partir de l'entretien retranscrit, la première étape a consisté à classer les informations afin de créer la légende de la carte. Bénédicte Michalon a construit un tableau permettant de répertorier les différentes étapes (types de lieux d'enfermement et moyens de transport utilisé entre deux lieux), la toponymie officielle de la ville et du pays dans lequel le lieu se situe, la durée de « séjour » dans le lieu (en heures, jours, semaines, mois, années), la période (en mois et année). L'objectif était ensuite "d'homogénéiser » les indications figurant dans le tableau afin d'avoir, pour chaque étape, le même type d'information. Nous avons également ajouté deux éléments. D'une part, la distance parcourue entre les lieux et, d'autre part, les aspects relationnels établis lors du parcours, qui s'effectue rarement seul (avec un passeur, avec d'autres personnes migrantes), et qui étaient évoqués dans le récit. Enfin, nous avons réalisé deux typologies: une première pour les lieux de confinement ${ }^{16}$; et une seconde pour le type de parcours (forcé ou volontaire).

Une fois la légende construite, la deuxième étape consistait à faire des choix concernant la représentation cartographique. Parmi les contraintes, il y avait celle des cartes relatives aux parcours des exilés qui devaient être dessinées à la main, pour mieux retranscrire l'intimité de l'expérience migratoire. Nous avons alors très vite abandonné l'idée d'un fond de carte conventionnel et opté pour la représentation des seuls pays traversés. D’abord, par 
souci de fidélité à ce qui avait été exprimé dans le récit et qui traduisait le vécu d'Ahmed, mais aussi pour des raisons d'échelles et d'emplacement sur la carte. Dans chacun des pays parcourus, il y avait plusieurs étapes qu'il aurait été difficile de cartographier si nous avions choisi un fond de carte de type planisphère. Puis concernant les figurés ${ }^{17}$, nous n'avons pas puisé dans une base de données existante, mais créé nos propres dessins afin qu'ils s'imposent d'emblée aux lecteurs et ainsi leur éviter de se référer trop souvent à la légende placée à côté de la carte.

À côté de la carte principale, nous avons inséré un graphique représentant le temps (en jours) passé dans les différentes structures : lieux de privation de liberté dépendant de l'État, lieux de privation de liberté dépendant des passeurs et centres d'accueil. En plus du titre de la carte, ce graphique permettait d'exprimer la temporalité du parcours, qui demeure souvent une dimension absente des cartographies sur le phénomène migratoire. Quant à la silhouette située en bas à gauche, elle avait été dessinée dans l'intention de personnifier le trait du parcours et de rappeler immédiatement au lecteur qu'il s'agissait avant toute chose de la vie d'un être humain. L’ensemble a ensuite été assemblé sur le logiciel Photoshop et passé en négatif, du trait noir sur fond blanc, au trait blanc sur fond noir, avant d'être remis entre les mains d'Étrange miroir. 


\section{De la carte statique à l'animation vidéo (Raphaël Rialland, Étrange miroir)}

Comme pour l'ensemble des cartes constituant l'exposition, notre apport s'est opéré en 4 étapes: identification du message et des informations essentielles à transmettre, traitement graphique, animation vidéo et création d'un paysage sonore accompagnant sa projection. Autrement dit, il s'agissait d'en faire un film, en proposant une narration et une poésie visuelle et sonore.

Pour cette carte dessinée par Lucie Bacon, nous avons conservé le dessin au trait blanc dans sa grande majorité et colorisé quelques éléments pour les valoriser (silhouette et graphique). Le graphiste, Guillaume Moitessier, a composé un arrière-plan en nuances sombres et orangées qui donne la tonalité de l'atlas du module 2 ("At the border ») dans lequel cette carte dessinée est projetée. L'animation permettra de donner vie à cette toile de fond sur laquelle ondulera un voile sombre pour donner la sensation de nuit, de voyage vers l'inconnu.

La mise en page au format de la table-atlas a nécessité plusieurs essais et allers-retours avec Lucie. Pour cette carte riche en détails, avec les contraintes de définition vidéo, nous avons dû optimiser la taille de la carte du parcours et de la légende, relativement fournie, afin d'en permettre la lecture. Ceci nous a contraints à supprimer certains éléments d'ornementation (des fils barbelés qui entouraient la carte figurant le parcours) faute de place et pour alléger la composition.

\section{La fabrique d'une œuvre poétique, visuelle et sonore}

Dans un second temps, l'animation vidéo a été utilisée pour dévoiler progressivement l'apparition des éléments de la carte. Le visiteur est guidé et peut suivre ce voyage étape par étape. À l'ouverture de la page, le personnage apparaît, accompagné successivement du titre et du fond de carte (contours des pays, étapes du parcours). Une petite boule de lumière jaune ambrée apparaît dans la silhouette, à l'emplacement du cœur, comme pour matérialiser lâme, la personnalité, l'individu ou la lumière intérieure. Cette lueur va ensuite retracer le parcours complexe effectué par Ahmed. Elle suit son tracé, marquant de courtes pauses aux endroits où il a séjourné, changeant d'allure en fonction des modes de transports empruntés. Les noms des pays et des villes du parcours apparaissent à son passage (Afghanistan, Kaboul, Russie, Moscou, Gomel, Biélorussie, etc.). Ce parcours, qui est fait d'innombrables bifurcations, stationnements et retours en arrière, a un caractère absurde, ahurissant.

Il nous semblait important de hiérarchiser ainsi l'arrivée des éléments. D'une part, parce que ce parcours labyrinthique a une grande force narrative en tant que tel. D'autre part, pour que l'on ait en tête le trajet avant d'appréhender la lecture analytique proposée par la légende. Celle-ci s'affiche donc dans un second temps et explicite les différentes étapes du voyage, ainsi que les entraves à l'origine de la sinuosité du parcours d'Ahmed.

Dans un troisième temps, le graphique apparaît de manière dynamique dans la marge à droite de l'Atlas. Il propose un troisième niveau de lecture, plus général, car il synthétise le temps passé dans chaque type de structure. Rompant avec la représentation horizontale traditionnelle du temps, les jours sont ici comptabilisés comme le seraient des marchandises. Cette donnée temporelle est la plus frappante. Elle outrepasse par sa démesure la complexité de l'itinéraire et fait référence à une dimension universelle : cinq ans d'une vie. Si l'on doit retenir quelque chose de ce voyage, c'est bien le fait qu'Ahmed a mis 5 ans à rejoindre les membres de sa famille vivant à Hambourg, comme l'indique le titre choisi par l'auteur. La lecture dictée par l'animation propose donc la chronologie de lecture suivante : (1) la narration/identification (on vit le parcours labyrinthique d'Ahmed); (2) l'analyse (la légende nous donne des informations sur les raisons de sa complexité) ; (3) la synthèse (on perçoit le voyage dans une temporalité universelle). La création du paysage sonore a constitué le lieu d'une interprétation poétique libre. S'imaginer dans la peau d'Ahmed attendant à un arrêt de bus 
la nuit, dans une zone en marge de la ville, ainsi la structure plastique de l'abribus vibre au passage des véhicules sur la chaussée détrempée. Par le mixage de sons concrets (ambiances extérieures, bruits de circulations, grincements de structures, goutte à goutte...), j'ai cherché à composer une photographie sonore de ce lieu tout en illustrant l'errance, la vie dehors, la sensation d'un voyage interminable, hors du temps, qui prend peu à peu une dimension surréaliste. Par touches éparses, la musique amène une douceur, pose un climat, une humanité en miroir de cet individu dont on suit le parcours sans entendre la voix.

\section{Conclusion}

Moving Beyond Borders propose une exploration sensible et sensée des parcours des migrants et des dispositifs responsables de leurs errances, dans le Sahara, en mer Méditerranée et/ou aux frontières orientales de l'Union européenne. Étrange miroir s'est emparée de la matière scientifique et militante fournie par Migreurop pour en faire un voyage immersif accessible au plus grand nombre. Moving Beyond Borders vise ainsi le partage de dix ans d'observations et de recherches sur les entraves, les injustices et les violations des droits des personnes migrantes. Elle entend aussi promouvoir une autre vision du monde, où la liberté de circulation serait garantie pour toutes et tous, et à ce titre constituer un vecteur de changement social au profit d'une société plus juste et plus équitable.

Le message que le réseau Migreurop s'évertue à faire passer depuis sa création, avec ses propres moyens d'action, a donc trouvé un véritable écho dans le travail plastique et sonore d'Étrange miroir. Imaginée comme une expérience sensible favorisant une vibration, une disposition à se laisser surprendre, cette exposition porte un message politique au travers d'une proposition artistique qui vient diversifier les manières dont on peut le percevoir, le ressentir, le rendre intelligible.

Moving Beyond Borders, qui s'inscrit dans une démarche militante et artistique, selon une approche multimédia, se caractérise aussi par sa vocation itinérante. Ainsi, cette exposition a déjà voyagé dans plusieurs villes de France : à Calais, Arcueil, Poitiers, Évry et Strasbourg. Elle a pu aussi circuler en Belgique à Bruxelles pour son inauguration, en Italie à Pozzallo dans le cadre du festival Sabir ou encore au Luxembourg au Centre de documentation sur les migrations humaines $(\mathrm{CDMH})$ à Dudelange. Elle a même traversé la Méditerranée pour faire escale à Rabat. 